\title{
Primary care patients in psychiatric clinical trials: a pilot study using videoconferencing Janet BW Williams*1,2, Amy Ellis ${ }^{1}$, Arthur Middleton ${ }^{3}$ and Kenneth A Kobak ${ }^{1}$
}

Address: ${ }^{1}$ MedAvante, Inc., MedAvante Research Institute, Hamilton, NJ, USA, ${ }^{2}$ Columbia University, Dept. of Psychiatry, New York, NY, USA and ${ }^{3}$ Hackensack University Medical Center, Hackensack, NJ, USA

Email: Janet BW Williams* - jwilliams@medavante.net; Amy Ellis - aellis@medavante.net; Arthur Middleton - amiddleton@humed.com; Kenneth A Kobak - kkobak@medavante.net

* Corresponding author

Published: 4 October 2007

Annals of General Psychiatry 2007, 6:24 doi:10.1186/1744-859X-6-24
Received: 26 July 2007

Accepted: 4 October 2007

This article is available from: http://www.annals-general-psychiatry.com/content/6/I/24

(c) 2007 Williams et al; licensee BioMed Central Ltd.

This is an Open Access article distributed under the terms of the Creative Commons Attribution License (http://creativecommons.org/licenses/by/2.0), which permits unrestricted use, distribution, and reproduction in any medium, provided the original work is properly cited.

\begin{abstract}
Background: While primary care physicians play a pivotal role in the treatment of depression, collaboration between primary care and psychiatry in clinical research has been limited. Primary care settings provide unique opportunities to improve the methodology of psychiatric clinical trials, by providing more generalizable and less treatment-resistant patients. We examined the feasibility of identifying, recruiting, screening and assessing primary care patients for psychiatric clinical trials using high-quality videoconferencing in a mock clinical trial.
\end{abstract}

Methods: I329 patients at two primary care clinics completed a self-report questionnaire. Those screening positive for major depression, panic, or generalized anxiety were given a diagnostic interview via videoconference. Those eligible were provided treatment as usual by their primary care physician, and had 6 weekly assessments by the off-site clinician via videoconferencing.

Results: 45 patients were enrolled over 22 weeks, with 36 (80\%) completing the six-week study with no more than two missed appointments. All diagnostic groups improved significantly; $94 \%$ reported they would participate again, $87 \%$ would recommend participation to others, $96 \%$ felt comfortable communicating via videoconference, and $94 \%$ were able to satisfactorily communicate their feelings via video.

Conclusion: Results showed that primary care patients will enroll, participate in and complete psychiatric research protocols using remote interviews conducted via videoconference.

\section{Background}

Primary care physicians have long been recognized as playing a pivotal role in the treatment of depression, providing the majority of all mental health treatments for this disorder $[1,2]$. They are often the first point of contact for patients with mental health concerns. It has been estimated that up to $22 \%$ of primary care patients have some form of co-morbid depression [3,4]. A collaborative model between primary care and mental health specialists that includes such elements as evidence-based treatment protocols, improved methods of screening and detection, patient education, and active monitoring of treatment adherence and outcome, has been shown to be an effective treatment intervention strategy[5-8]. 
While there has been increased focus on the collaboration between primary care and mental health practitioners in the clinical care of patients, less attention has been paid to collaboration between the two disciplines in clinical research. Primary care settings provide unique opportunities to improve the methodology of clinical trials. Traditionally, clinical trials in depression primarily recruit through newspaper ads, resulting in a high proportion of treatment-resistant and atypical patients. These patients are often more likely to fail treatment, increasing the chances of a failed trial. Primary care patients are more likely to be treatment naïve, as depression is often first identified in a primary care setting. In addition, patients treated in a primary care setting may be less likely to be lost to follow-up, as they usually already have an ongoing relationship with the treatment provider. There is some evidence that primary care patients may also be less likely to respond to placebo: in a series of four generalized anxiety disorder studies, a $20 \%$ higher placebo response rate was found in patients recruited from psychiatric settings as compared to primary care sites [9]. The high prevalence rate of depression and other mental health disorders in primary care can also help facilitate patient recruitment for clinical trials.

An obstacle to participation in clinical depression trials by primary care physicians is the lack of formal training in the diagnosis and assessment of depression using standardized rating scales. Other barriers include a lack of experience in research methodology, and limited time for clinical trial management. One potential solution to this problem is the use of off-site expert, centralized raters that are linked to the various study sites through videoconferencing or teleconferencing [10]. These raters can remotely administer the primary outcome measures to study patients during their regularly scheduled study visits. Centralized raters have several advantages, including improved reliability and quality, more thorough calibration and monitoring for interview quality, and reduced rating bias (as they are independent from the study site). Centralized raters can also be blinded to study visit, study design, and study entrance requirements, further reducing expectation biases. Several studies have shown that rating scales administered remotely by videoconference or teleconference yield equivalent results as when administered face-to-face [11-20].

The current study examined the feasibility of identifying, recruiting and screening primary care patients for clinical trials, and examined patient comfort, satisfaction, and adherence in a mock clinical trial using high-quality videoconferencing for screening and ongoing evaluations by remote raters.

\section{Methods}

Patients at two large research-naïve primary care practices were provided a letter at check-in from their primary care physician inviting them to participate in a study of the usefulness of telemedicine in evaluating patient symptoms. Those who were interested in participating completed a self-report questionnaire (the Patient Health Questionnaire; PHQ) [21] in the waiting room to assess their eligibility. The PHQ screens for common mental health disorders found in primary care patients. Those screening positive for major depressive disorder (MDD), generalized anxiety disorder (GAD), or panic disorder (PD) on the PHQ and who were otherwise eligible to participate (i.e. not currently receiving mental health treatment such as psychotherapy or pharmacotherapy, not currently abusing alcohol or drugs, or having suicidal ideation) were scheduled for a diagnostic interview conducted remotely by a well-trained rater using high-quality videoconferencing. The diagnostic interview contained the overview from the Structured Clinical Interview for DSM-IV (SCID) [22] and diagnostic questions from the PHQ. Patients whose positive screen was confirmed by the diagnostic interview were also administered a symptom rating scale: either the Hamilton Depression Rating Scale (HAMD) [23], Hamilton Anxiety Rating Scale (HAMA) [24], or Panic Disorder Severity Scale (PDSS) [25], depending on their primary diagnosis (MDD, GAD, or PD respectively). Structured interview guides were used to administer all rating scales [25-27]. Patients were asked to come back once a week for 6 weeks for follow-up evaluations, as if they were in a therapeutic clinical trial. At their weekly visits, patients were evaluated remotely with either the HAMD, HAMA, or PDSS, as well as the Clinical Global Impressions of Change Scale (CGI-C) [28].

The results of the initial diagnostic interview were shared with the patient's primary care physician. The physician decided, based on this information and his or her knowledge of the patient, whether treatment with medication should be offered or whether another intervention or referral was indicated. Regardless of whether the patient was offered or accepted a medication treatment plan, the patient was invited to participate in the longitudinal tracking phase.

Throughout the study, the data regarding treatment response was available to the treating physician. If the patient's condition deteriorated significantly, the interviewer notified the primary care physician, who followed agreed-upon next steps. A 25\% increase in the HAM-D, HAM-A, PDSS score, suicidal ideation or any condition requiring hospitalization mandated withdrawal from the study and immediate treatment (for those not already receiving treatment). At the end of the study, a copy of the 
research evaluations was made for inclusion in the patient's medical chart.

Patients completed a survey after their first and last remote assessment in which they were asked to rate their overall preferences for the videoconferencing process, their level of agreement with descriptions of various attributes of their experience, and to respond to three open-ended questions probing attitudes towards remote assessment.

The study was approved by the New York State Psychiatric Institute Institutional Review Board, and all patients signed informed consent statements. Patients received $\$ 100$ for their participation. Patients who discontinued early were paid on a pro-rata basis for number of visits completed.

\section{Telemedicine equipment and procedure}

Remote interviews were conducted using H.323 IP standards-based Polycom iPower Videoconferencing Systems (Polycom, Inc., Pleasanton, CA), connected using ISDN lines (i.e., multiple dedicated phone lines that can handle voice, video and data). The system ran at an industry standard bit rate of $384 \mathrm{kps}$, was HIPAA compliant, and occurred via a secure encrypted connection. Interviews were conducted in a private room with a desk and a door for privacy. The on-site research coordinator oriented the patient on what to do and where to go when the interview was completed, and answered any questions the patient had. The research coordinator waited with the patient to answer the call from the remote interviewer, then left the room and closed the door to ensure the patient's complete privacy.

\section{Results \\ Study flow}

A total of 1329 patients were screened in the waiting room with the PHQ. Of these, 211 patients (16\%) screened positive for one of the three mental health disorders on the PHQ. Of these 211, $76(36 \%)$ met one of the exclusion criteria and 56 (27\%) declined consent, leaving 79 (37\%) patients eligible for participation in the study. Of the 79 consenting patients, 9 were lost to follow-up between consent and the initial remote assessment, leaving 70 subjects who were administered the initial follow-up diagnostic interview by the remote clinician. Of the 70 patients who were available for follow-up, 15 were found to have only subthreshold diagnoses, and 10 were found to have met other exclusionary criteria, leaving 45 patients eligible for the tracking phase of the study.

Enrollment by diagnosis and study site is presented in Table 1. Of the 45 patients enrolled, 17 (38\%) had MDD as the primary diagnosis, $14(31 \%)$ had GAD, and 14
Table I: Patient enrollment by diagnosis and study site

\begin{tabular}{llll}
\hline Study arm & Site I & Site II & Total study \\
\hline Enrollment target & 10 & 30 & \\
Actual enrollment & 12 & 33 & $45(100 \%)$ \\
Major depression & 7 & 10 & $17(38 \%)$ \\
Generalized anxiety & 1 & 13 & $14(31 \%)$ \\
Panic & 4 & 10 & $14(31 \%)$ \\
Total & 12 & 33 & $45(100 \%)$ \\
& & &
\end{tabular}

(31\%) had panic disorder. The proportion of GAD patients vs PD patients in the GAD/PD arm was naturalistic and no attempt to balance to a pre-determined level was made.

The total time taken to enroll these 45 eligible patients was 22 weeks. Site 1 had a target enrollment of 10 patients, and enrolled 12 patients over 8.4 weeks, and site 2 had a target enrollment of 30 patients, and enrolled 33 patients over 16.6 weeks. There was a 3-week overlap of enrollment time between the sites. Given the staggered enrollment period, the total enrollment rate was approximately two eligible patients per week.

\section{Study adherence}

Of the 45 patients enrolled, 36 (80\%) completed the sixweek study with no more than two missed appointments. A total of 27 patients $(60 \%)$ completed the entire series of six visits, 7 patients $(16 \%)$ completed with one missed interview, 2 patients $(4 \%)$ completed with two missed interviews, and 9 patients (20\%) dropped out.

Reasons for the 9 drop-outs were: patient did not return call (4), phone disconnected (1), parent developed cancer (1), too many medical problems (1), looking for therapy ('not getting anything out of assessments') (1), and patient denied being depressed (1).

There were no significant differences between those completing at least $80 \%$ of visits, and those who did not in terms of baseline symptom severity or gender; however, those who completed were significantly older than those who did not ( 47 vs 34 years, $\mathrm{p}=0.022$ ). In addition, a greater percentage of completers (17 of 24) than noncompleters ( 3 of 9 ) agreed with the statement 'I would prefer to be interviewed in my doctors office using this technology than have to travel to be interviewed by someone face-to-face', $\chi^{2}(1)=3.855, \mathrm{p}=0.049$.

\section{Clinical outcomes}

Data on patients' weekly scores are presented in Table 2. All three cohorts of patients improved significantly over time, although no data were collected on what treatments they received. The mean change for depressed patients on 
Table 2: Mean scores on HAMD, HAMA and PDDS by study visit

\begin{tabular}{llllllll}
\hline & Baseline & Week I & Week 2 & Week 3 & Week 4 & Week 5 & Week 6 \\
\hline Depression (HAMD) & 14.71 & 11.06 & 9.25 & 7.69 & 6.50 & 5.13 & 4.56 \\
GAD (HAMA) & 20.57 & 19.25 & 12.64 & 14.64 & 13.71 & 14.86 & 13.14 \\
Panic disorder (PDSS) & 12.14 & 7.14 & 5.36 & 5.29 & 5.00 & 6.21 & 5.50 \\
\hline
\end{tabular}

the HAMD was 7.43 points (from 20.57 to 13.14 ), $t(13)$ $=2.67, \mathrm{p}=0.019$. The mean change for GAD patients on the HAMA was 10.06 points (from 14.63 to 4.56 ), $\mathrm{t}(15)=$ $4.48, \mathrm{p}<0.001$, and the mean change for patients with panic disorder was 6.64 points on the PDDS (from 12.14 to 5.50$), t(13)=3.198, \mathrm{p}=0.007$. Average time per assessment was $19.75 \mathrm{~min}$ for the HAMD, $21.43 \mathrm{~min}$ for the HAMA, and 17.45 min for the PDDS.

\section{Patient and physician satisfaction}

Satisfaction and comfort levels of the participating patients using telemedicine were very high (Table 3). A total of $94 \%$ indicated that they would be 'somewhat likely' or 'very likely' to participate again, and $86 \%$ said they would be 'somewhat likely' or 'very likely' to recommend to others participation in clinical studies using videoconferencing. Only one participant rated the experience 'somewhat negative', with the rest feeling either somewhat or very positive $(91 \%)$ or neutral $(7 \%)$. A total of $96 \%$ reported feeling comfortable talking to a person on a TV monitor, and $93 \%$ agreed that they were able to satisfactorily communicate their feelings to the interviewer. Although 38\% somewhat or strongly agreed that they preferred to be interviewed by someone face-to-face, it is notable that $62 \%$ either did not agree, or neither agreed nor disagreed. Indeed, $89 \%$ agreed that they 'felt like [they were] talking to someone in the same room.' A total of $60 \%$ agreed that they would prefer to be interviewed in their doctor's office using this technology rather than have to travel to be interviewed by someone face-to-face.

Primary care physicians and staff had an average positive rating of 4.8 (out of 5) when asked the question, 'Would you be likely to participate in a telemedicine study again?' (Response options ranged from 1 (very unlikely) to 5 (very likely)).

\section{Discussion}

Results of this study support the hypothesis that patients in primary care settings will enroll, participate in and complete research protocols using remote interviews conducted via videoconference. The waiting room screening process was time-efficient, accepted by patients, and resulted in an adequate yield of potential subjects. The $20 \%$ drop-out rate compares favorably to the drop-out rate found in clinical trials conducted at psychiatry sites $[29,30]$. A secondary positive outcome of this process is the identification of patients with mental disorders that may otherwise have gone undetected and untreated.

To facilitate patient participation in future studies with remote assessors, sites must foster a patient-friendly environment and ensure specific compensation for the site staff to align their incentives with that of the physician's practice. In addition, patient compensation may have played a factor in the completion rate. It is possible that the completion rate may have been less if patients received no compensation. The study process could have been improved by adding questions to the PHQ that screened out patients who did not meet other inclusion criteria (e.g., currently in treatment, etc.). For Site II enrollment, just such an amendment to the PHQ was provided and only a single patient who did not fit the inclusion/ exclusion criteria made it through to the Phase II screening interview. Patient declines may also have been minimized by a shorter time window between screening and follow-up contact. Delay in scoring the PHQ and notifying patients that they qualified for the study may have resulted in lowering the participation rate. In the future, incorporating the informed consent process in the videoconference procedure may further enhance the ability to enroll patients. Dobscha and colleagues [31] successfully utilized this method of obtaining consent in a study of depressed primary care patients, and found patient satisfaction and acceptance was high, with no increase in patients lost to follow up.

In summary, the current study provides support for the use of primary care sites in clinical drug trials in psychiatry. Using primary care sites may help solve problems with patient recruitment, as well as overcoming some of the methodological problems associated with patients recruited in psychiatric settings. The use of independent raters has been shown to further improve clinical trial methodology by improving reliability and quality of assessments, decreasing bias, and, in a recent study, decreasing placebo response [32]. The combination of centralized independent raters and primary care settings should provide a powerful new approach to the conduct of clinical trials. 
Table 3: Patient satisfaction survey

\begin{tabular}{|c|c|c|c|c|c|}
\hline & Very negative & Somewhat negative & Neutral & Somewhat positive & Very positive \\
\hline $\begin{array}{l}\text { I. How would you describe your overall } \\
\text { experience with the study? }\end{array}$ & $0(0 \%)$ & I (2\%) & $3(7 \%)$ & 17 (38\%) & $24(53 \%)$ \\
\hline & Very unlikely & Somewhat unlikely & Neither likely nor unlikely & Somewhat likely & Very likely \\
\hline $\begin{array}{l}\text { 2. Would you be likely to recommend to } \\
\text { a friend to participate in clinical studies } \\
\text { using videoconferencing? }\end{array}$ & $0(0 \%)$ & $\mathrm{I}(2 \%)$ & $5(11 \%)$ & $10(22 \%)$ & $29(65 \%)$ \\
\hline $\begin{array}{l}\text { 3. Would you participate in another study } \\
\text { using this technology? }\end{array}$ & I (2\%) & I (2\%) & I (2\%) & $7(16 \%)$ & $35(78 \%)$ \\
\hline
\end{tabular}

Strongly disagree Somewhat disagree Neither agree nor disagree $\quad$ Somewhat agree $\quad$ Strongly agree

\begin{tabular}{|c|c|c|c|c|c|}
\hline $\begin{array}{l}\text { I. I was comfortable talking to a person } \\
\text { on a TV monitor }\end{array}$ & $0(0 \%)$ & $\mathrm{I}(2 \%)$ & $\mathrm{I}(2 \%)$ & $15(33 \%)$ & $28(63 \%)$ \\
\hline $\begin{array}{l}\text { 2. I was able to satisfactorily communicate } \\
\text { my feelings to the interviewer }\end{array}$ & $0(0 \%)$ & $2(4 \%)$ & I (2\%) & $8(18 \%)$ & $34(76 \%)$ \\
\hline $\begin{array}{l}\text { 3. Videoconferencing/teleconferencing is a } \\
\text { good way to get a psychological } \\
\text { evaluation }\end{array}$ & $0(0 \%)$ & I (2\%) & $6(13 \%)$ & $12(27 \%)$ & $26(58 \%)$ \\
\hline $\begin{array}{l}\text { 4. The scheduling was flexible enough for } \\
\text { me }\end{array}$ & $0(0 \%)$ & $2(5 \%)$ & $\mathrm{I}(2 \%)$ & $6(13 \%)$ & $36(80 \%)$ \\
\hline $\begin{array}{l}\text { 5. I was comfortable that the interviews } \\
\text { were kept confidential }\end{array}$ & $0(0 \%)$ & $\mathrm{I}(2 \%)$ & $0(0 \%)$ & $8(18 \%)$ & $36(80 \%)$ \\
\hline $\begin{array}{l}\text { 6. I would prefer to be interviewed by } \\
\text { someone face to face }\end{array}$ & $3(7 \%)$ & $6(13 \%)$ & $19(42 \%)$ & $13(29 \%)$ & $4(9 \%)$ \\
\hline $\begin{array}{l}\text { 7. I established a good relationship with } \\
\text { my interviewer }\end{array}$ & $0(0 \%)$ & $\mathrm{I}(2 \%)$ & $8(I 8 \%)$ & $19(42 \%)$ & $17(38 \%)$ \\
\hline $\begin{array}{l}\text { 8. I was able to see the interviewer } \\
\text { satisfactorily }\end{array}$ & $0(0 \%)$ & I (2\%) & $0(0 \%)$ & $9(20 \%)$ & $35(78 \%)$ \\
\hline $\begin{array}{l}\text { 9. I would prefer to be interviewed in my } \\
\text { doctor's office using this technology than } \\
\text { have to travel to be interviewed by } \\
\text { someone face-to-face }\end{array}$ & $2(4 \%)$ & $4(9 \%)$ & $12(27 \%)$ & $8(18 \%)$ & $19(42 \%)$ \\
\hline 10. I was comfortable in the room alone & $0(0 \%)$ & I (2\%) & $0(0 \%)$ & $7(16 \%)$ & $37(82 \%)$ \\
\hline $\begin{array}{l}\text { II. It felt like I was talking to someone in } \\
\text { the same room }\end{array}$ & $0(0 \%)$ & $2(4 \%)$ & $3(7 \%)$ & $9(20 \%)$ & $31(69 \%)$ \\
\hline $\begin{array}{l}\text { 12. There were technical problems } \\
\text { relating to the sound or image quality }\end{array}$ & $33(73 \%)$ & $4(9 \%)$ & $4(9 \%)$ & $2(4 \%)$ & $2(4 \%)$ \\
\hline $\begin{array}{l}\text { 13. How good was the quality of the } \\
\text { image? }\end{array}$ & $0(0 \%)$ & $\mathrm{I}(2 \%)$ & $2(4 \%)$ & 17 (38\%) & $25(56 \%)$ \\
\hline \multirow[t]{2}{*}{$\begin{array}{l}\text { 14. How good was the quality of the } \\
\text { sound? }\end{array}$} & $0(0 \%)$ & $0(0 \%)$ & $\mathrm{I}(2 \%)$ & $13(29 \%)$ & $31(69 \%)$ \\
\hline & Never & Almost never & Occasionally & Often & Very often \\
\hline $\begin{array}{l}\text { 15. Were the sessions interrupted by } \\
\text { technical difficulties? }\end{array}$ & $36(82 \%)$ & $5(12 \%)$ & $\mathrm{I}(2 \%)$ & I (2\%) & $\mathrm{I}(2 \%)$ \\
\hline 16. How often do you use a computer? & $6(13 \%)$ & $3(7 \%)$ & II (24\%) & $8(18 \%)$ & $17(38 \%)$ \\
\hline $\begin{array}{l}\text { 17. Before the study, how often did you } \\
\text { use videoconferencing? }\end{array}$ & 38 (85\%) & $2(5 \%)$ & $2(5 \%)$ & $2(5 \%)$ & $0(0 \%)$ \\
\hline
\end{tabular}

\section{Competing interests}

JBWW, KAK and AE are employed by MedAvante, a company that provides centralized rating services for clinical trials.

\section{Authors' contributions}

JBWW and AM conducted the clinical interviews, and helped with study design. AE helped with study design.
KK conducted the statistical analyses and drafted the manuscript.

\section{Acknowledgements}

We would like to acknowledge the contributions of Cindy Aaronson and Heather Goldman who conducted clinical interviews, and Angela Wilmer for project management. 


\section{References}

I. Shapiro S, Skinner EA, Kessler LG, Von Korff M, German PS, Tischler GL, Leaf PJ, Benham L, Cottler L, Regier DA: Utilization of health and mental health services: three epidemiologic catchment area sites. Archives of General Psychiatry 1984, 41:971-978.

2. Regier DA, Goldberg ID, Taube CA: The de facto US mental health services system: a public health perspective. Archives of General Psychiatry 1978, 35:685-693.

3. Barrett JE, Barrett JA, Oxman TE, Gerber PD: The prevalence of psychiatric disorders in a primary care practice. Archives of General Psychiatry 1988, 45: I I00-II06.

4. Coyne JC, Fechner-Bates S, Schwenk TL: Prevalence, nature, and comorbidity of depressive disorders in primary care. Gen Hosp Psychiatry 1994, I6(4):267-276.

5. Simon G: Collaborative care for depression. Bmj 2006, 332(7536):249-250.

6. Katzelnick D, Simon G, Pearson S, Manning W, Kobak K: Depression management programs. Arch Fam Med 2000, 9(8):689-670.

7. Katon W, von Korff MV, Lin E, Walker E, Simon GE, Bush T, Robinson $P$ : Collaborative management to achieve treatment guidelines. Impact on depression in primary care. Journal of the American Medical Association 1995, 273(13): I026-1031.

8. Simon GE, VonKorff M, Rutter C, Wagner E: Randomised trial of monitoring, feedback, and management of care by telephone to improve treatment of depression in primary care. BMJ 2000, 320:550-554.

9. Schweizer E, Rickels K: Placebo response in generalized anxiety: its effect on the outcome of clinical trials. J Clin Psychiatry 1997, 58 Suppl I I:30-38.

10. Kobak KA, Kane JM, Thase ME, Nierenberg AA: Why Do Clinical Trials Fail?: The Problem of Measurement Error in Clinical Trials: Time to Test New Paradigms? J Clin Psychopharmacol 2007, 27(I): I-5.

II. Kobak KA: A comparison of face-to-face and videoconference administration of the Hamilton Depression Rating Scale. J Telemed Telecare 2004, I0(4):23 I-235.

12. Baer L, Cukor P, Jenike MA, Leahy L, O'Laughlen J, Coyle JT: Pilot studies of telemedicine for patients with obsessive-compulsive disorder. Am J Psychiatry 1995, I 52(9): I 383-I385.

13. Menon AS, Kondapavalru P, Krishna P, Chrismer JB, Raskin A, Hebel $J R$, Ruskin PE: Evaluation of a portable low cost videophone system in the assessment of depressive symptoms and cognitive function in elderly medically ill veterans. J Nerv Ment Dis 200I, I 89(6):399-40I.

14. Zarate CA Jr., Weinstock L, Cukor P, Morabito C, Leahy L, Burns C, Baer L: Applicability of telemedicine for assessing patients with schizophrenia: acceptance and reliability. J Clin Psychiatry 1997, 58(I):22-25.

15. Hyler SE, Gangure DP, Batchelder ST: Can telepsychiatry replace in-person psychiatric assessments? A review and meta-analysis of comparison studies. CNS Spectr 2005, I 0(5):403-4I3.

16. Simon GE, Revicki D, von Korff M: Telephone assessment of depression severity. Journal of Psychiatric Research 1993, 27(3):247-252.

17. Aneshensel CS, Frerichs RR, Clark VA, Yokopenic PA: Measuring depression in the community: a comparison of telephone and personal interviews. Public Opin $Q 1982,46$ (I): II $10-121$.

18. Rohde P, Lewinsohn PM, Seeley JR: Comparability of telephone and face-to-face interviews in assessing axis I and II disorders. American Journal of Psychiatry 1997, I 54:1593-1598.

19. Pinto-Meza A, Serrano-Blanco A, Penarrubia MT, Blanco E, Haro JM: Assessing depression in primary care with the PHQ-9: can it be carried out over the telephone? J Gen Intern Med 2005, 20(8):738-742.

20. Williams JBW, Kobak KA: Development and Reliability of the SIGMA: A structured interview guide for the MontgomeryAsberg Depression Rating Scale (MADRS). Br J Psychiatry in press.

21. Spitzer RL, Kroenke K, Williams JB: Validation and utility of a selfreport version of PRIME-MD: the PHQ primary care study. Jama 1999, 282( I 8): I 737-I744.

22. Spitzer RL, Williams JBW, Gibbon M, First MB: The Structured Clinical Interview for DSM-III-R (SCID) I: history, rationale and description. Archives of General Psychiatry 1992, 49:624-629.

23. Hamilton M: A rating scale for depression. Journal of Neurology, Neurosurgery and Psychiatry 1960, 23:56-62.
24. Hamilton $M$ : The assessment of anxiety states by rating. British Journal of Medical Psychology 1959, 32:50-55.

25. Shear KM, Brown TA, Barlow DH, Money R, Sholomskas DE, Woods SW, Gorman JM, Papp L: Multicenter collaborative panic disorder severity scale. American Journal of Psychiatry 1997, I54( I I): I57|-| 575 .

26. Williams JBW: A structured interview guide for the Hamilton Depression Rating Scale. Archives of General Psychiatry 1988, 45:742-747.

27. Williams JBW: Structured Interview Guide for the Hamilton Anxiety Scale (SIGH-A). New York, NY, New York State Psychiatric Institute; 1996.

28. Guy W: ECDEU Assessment Manual for Psychopharmacology, revised. Rockville, MD, National Institute of Mental Health, US Dept. of Health, Education, and Welfare publication ADM 76-338.; 1976.

29. Livingston MG, Livingston HM: New antidepressants for old people? BMJ 1999, 3 I 8(7 I 99): I640- I64I.

30. MacGillivray S, Arroll B, Hatcher S, Ogston S, Reid I, Sullivan F, Williams B, Crombie I: Efficacy and tolerability of selective serotonin reuptake inhibitors compared with tricyclic antidepressants in depression treated in primary care: systematic review and meta-analysis. $B m j$ 2003, 326(7397): 1014.

3I. Dobscha SK, Corson K, Solodky J, Gerrity MS: Use of videoconferencing for depression research: enrollment, retention, and patient satisfaction. Telemed J E Health 2005, I I ( I):84-89.

32. Kobak KA, DeBrota DJ, Engelhart N, Williams JBW: Site vs. Centralized Raters in a Clinical Depression Trial: Boca Raton, FL. ; 2006.
Publish with Bio Med Central and every scientist can read your work free of charge

"BioMed Central will be the most significant development for disseminating the results of biomedical research in our lifetime. "

Sir Paul Nurse, Cancer Research UK

Your research papers will be:

- available free of charge to the entire biomedical community

- peer reviewed and published immediately upon acceptance

- cited in PubMed and archived on PubMed Central

- yours - you keep the copyright

Submit your manuscript here:

http://www.biomedcentral.com/info/publishing_adv.asp
BioMedcentral 